\title{
RECENT PRECISION EXPERIMENTS WITH EXOTIC NUCLEI PRODUCED WITH URANIUM PROJECTILES AND EXPERIMEN- TAL PROSPECTS AT FAIR
}

\author{
H. Geissel ${ }^{1,2, a}$, L. Chen ${ }^{2}$, T. Dickel ${ }^{1}$, F. Farinon ${ }^{1}$, I. Dillmann ${ }^{1,2}$, R. Knöbel ${ }^{1}$, J. Kurcewicz ${ }^{1}$, \\ I. Mukha ${ }^{1}$, G. Münzenberg ${ }^{1}$, C. Nociforo ${ }^{1}$, Z. Patyk ${ }^{1}$, S. Pietri ${ }^{1}$, W.R. Plass ${ }^{1,2}$, A. Prochazka ${ }^{1}$, \\ C. Scheidenberger ${ }^{1,2}$, M. Takechi ${ }^{1}$, H. Weick ${ }^{1}$, J.S. Winfield ${ }^{1}$, and M. Winkler ${ }^{1}$ \\ ${ }^{1}$ GSI Helmholtzzentrum für Schwerionenforschung GmbH, 64291 Darmstadt, Germany \\ 2II. Physikalisches Institut, Universität Giessen, 35392 Giessen, Germany
}

\begin{abstract}
.
Precision experiments with relativistic fragments separated in-flight require special experimental methods to overcome the inherent large emittance from the creation in nuclear reactions and atomic interactions in matter. At GSI relativistic exotic nuclei have been produced via uranium projectile fragmentation and fission and investigated with the inflight separator FRS directly, or in combination with either the storage-cooler ring ESR or the FRS Ion Catcher. $1000 \mathrm{~A} \cdot \mathrm{MeV}{ }^{238} \mathrm{U}$ ions were used to create 60 new neutron-rich isotopes separated and identified with the FRS to measure their production cross sections. In another experimental campaign the fragments were separated in flight and injected into the storage-cooler ring ESR for accurate mass and lifetime measurements. In these experiments we have obtained accurate new mass values analyzed via a novel method which has reduced the systematic errors for both Schottky Mass Spectrometry (SMS) and for Isochronous Mass Spectrometry (IMS). Pioneering experiments have been carried out with the FRS Ion Catcher consisting of three experimental components, the dispersive magnetic system of the FRS with a monoenergetic and a homogeneous degrader, a cryogenic stopping cell filled with pure helium and a multiple-reflection time-of flight mass separator. The FRS Ion Catcher enables high precision spectroscopy experiments with $\mathrm{eV}$ to $\mathrm{keV}$ exotic nuclides. Results from these different FRS experiments are presented in this overview together with prospects for the next-generation facility Super-FRS. The novel features of the Super-FRS compared with the present FRS will be discussed in addition.
\end{abstract}

\section{Experimental conditions and setups}

Heavy neutron-rich nuclei in the neighborhood of and at the $\mathrm{N}=126$ shell closure are of great interest for nuclear spectroscopy and astrophysics [1]. Experimentally it is a challenge to produce and identify these nuclei because of the small production cross sections, short lifetimes, and difficult separation in flight due broad charge-state distributions [2].

a e-mail: h.geissel@gsi.de

This is an Open Access article distributed under the terms of the Creative Commons Attribution License 2.0, which permits unrestricted use, distribution, and reproduction in any medium, provided the original work is properly cited. 
The stochastic collision processes which generate the interesting nuclides have the disadvantage that the reaction products populate a large phase space. The resulting large longitudinal and transverse emittance of the beam strongly limits high-resolution measurements. There are several experimental solutions to circumvent this inevitable property. One way of improvement is, for example, the generation of exotic nuclei at high kinetic energies because the phase-space enlargement scales inversely with the momentum of the reaction products and furthermore the population in different ionic states can be dramatically reduced as well. Therefore, the corresponding in-flight facilities aim at high kinetic energies to reach preferably the velocities where all fragments up to uranium emerge fully ionized from the production target.

Another way to reach experimental conditions for precision experiments with exotic nuclides is to reduce the phase space via cooling. The fragments spatially separated by in-flight facilities inject the fragments of interest into a storage-cooler ring [3-5]. Cooling in storage rings have been successfully applied to reach ideal conditions for precise revolution time measurements of the circulating ions. For example, applying electron cooling a relative velocity spread down to $10^{-7}$ was reached an ideal condition to perform mass and lifetime measurements of the stored exotic nuclei. The reduction of the phase space can also be reached by slowing down of an energy bunched beam in a gas-filled stopping cell [6-8]. In the latter method it is required that the relativistically produced and separated beam penetrates through a shaped degrader positioned in a dispersive focal plane of an ion-optical device. The efficiency of this method is strongly depend on the momentum resolution of the ionoptical spectrometer, the degrader shape and homogeneity and of course on the incident kinetic energy [9]. The energy determines the absolute value of the resulting range straggling and has to be selected as low as possible but such that the in-flight separation is not hampered by the appearance of ionic charge states. Besides these primary conditions it must be also the aim to have a large areal density and an excellent extraction efficiency and time of the gas-filled stopping cell.

There are experiments where the phase-space reduction is not possible e.g., because of the lifetime of the species with respect to the cooling time. In this case one can apply ion-optical methods to circumvent the influence of the inevitably large phase space. An elegant solution is to perform experiments with dispersion-matched spectrometers [10] or use longitudinal dispersive systems like a storage ring in the isochronous operating mode [4].

In this overview article we illustrate with recent experiments performed at GSI with the fragment separator FRS and its combinations with the storage ring ESR or with a monoenergetic degrader and the gas-filled ion catcher (IC). The prospects of such research at the international facility for antiprotons and ions FAIR will be briefly outlined in the last chapter of this review.

\section{Discovery of New Neutron-Rich Isotopes}

A $1000 \mathrm{~A} \cdot \mathrm{MeV}^{238} \mathrm{U}$ projectile beam with an intensity of $10^{9} / \mathrm{s}$ impinged on a $1.6 \mathrm{~g} / \mathrm{cm}^{2}$ thick beryllium target placed at the entrance of the FRS $[2,11]$. The heavy reaction products $\mathrm{Z}>60$ were separated with the FRS operated in an overall achromatic ion-optical mode. Two degraders, located at the first (F1) and second (F2) focal planes were used, see figure 1. In this way, the reaction products are spatially separated. The complete particle identification in-flight was performed on an event-by-event basis with time-of-flight, energy-deposition and magnetic rigidity measurements.

In this way an unambiguous isotope identification was achieved. Only bare isotopes have been considered in the cross section determination, the contributions from other charge states have been calculated and taken into account [12]. The ion-optical transmission has been calculated by using the Monte-Carlo simulation program MOCADI [13] taking into account the different kinematics of projectile fragmentation and fission. The contribution of each reaction type was determined for the 
a)

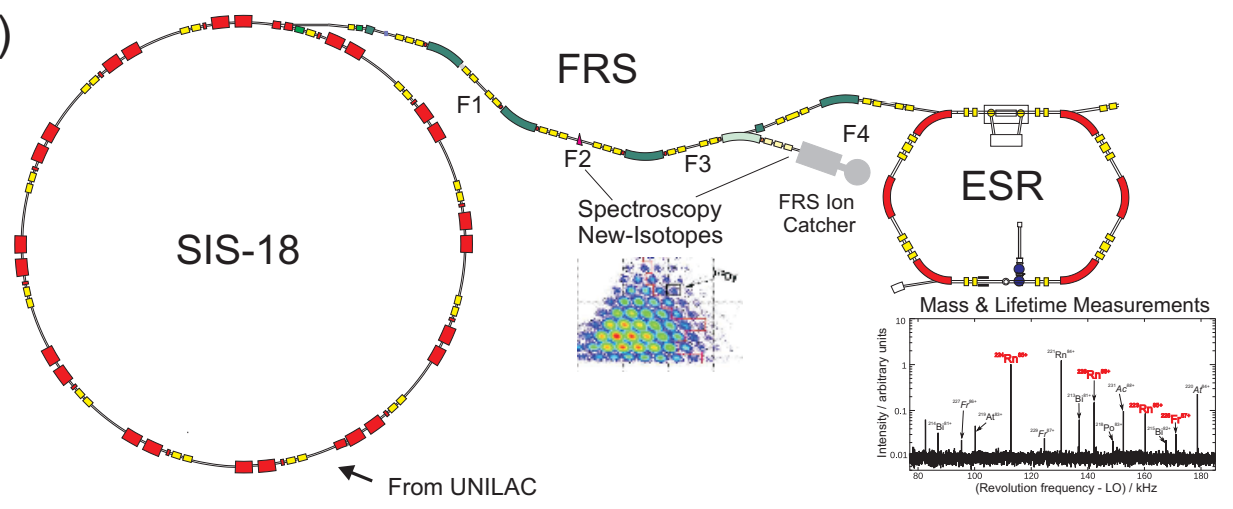

b)

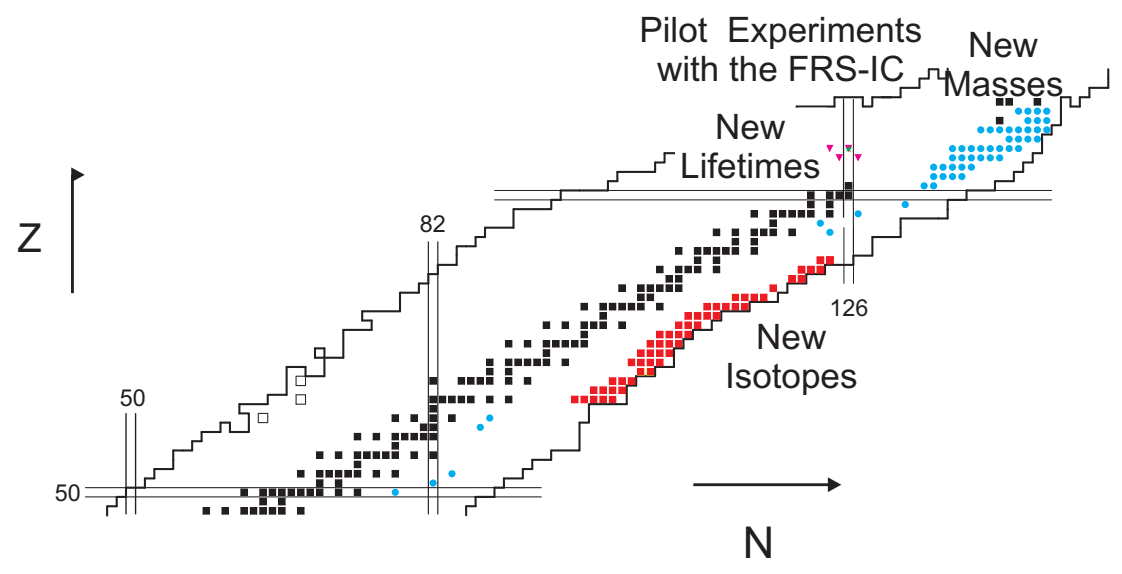

Figure 1. (a): Schematic view of the experimental facilities where the different experiments described in this proceedings have been performed. (b): Chart of nuclides with the new isotopes and those which have been investigated in the mass measurement, lifetime investigation and FRS Ion Catcher commissioning.

observed nuclides by the ABRABLA code [14]. In Figure 3 an example is shown for the measured Sm and Pt isotopes [2] compared with different theoretical predictions. Results from a previous FRS experiment using a hydrogen production are included (open circles) [15]. The difference in the overlapping isotopes might be caused by the different excitation energies in the different target materials. For the most neutron-rich isotopes of europium covered in this experiment the fission reaction completely dominates the production, whereas for platinum fragmentation dominates and fission is negligible. The ABRABLA predictions show an overall good agreement with the experimental data measured in the present work. Based on the results of ABRABLA calculations, we can state that the observed new neutron-rich isotopes of the elements between $60 \leq \mathrm{Z} \leq 66$ are produced mainly by fission, while fragmentation plays the major role in the production of the isotopes of the elements above $Z=72$. For the most neutron-rich nuclei, predictions of the analytical COFRA [16] code have been used for the range of elements where fragmentation is dominant. The COFRA model is in general also in good agreement with the experimental data, although gradual deviations from the experimental results can be observed for the heaviest (Os-Pt) nuclei. The EPAX-3 predictions are also quite good in the region 

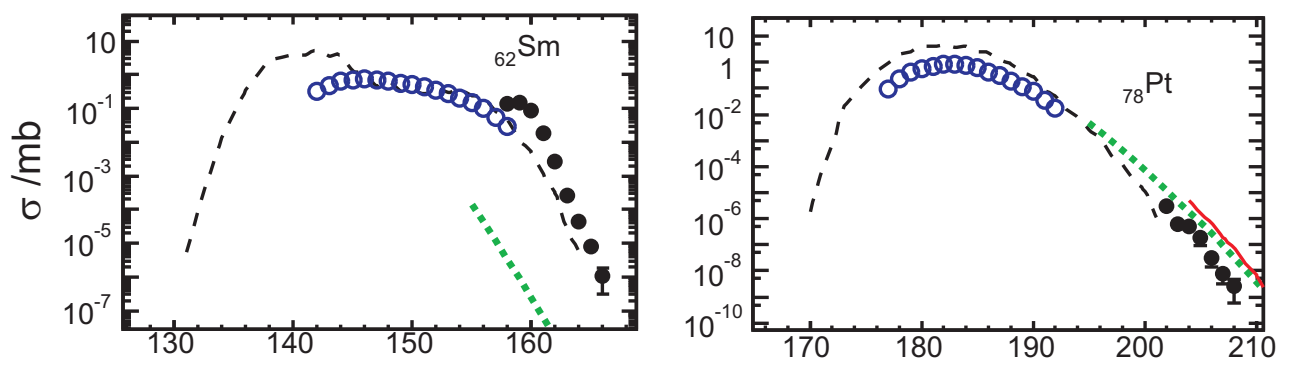

\section{Mass Number}

Figure 2. Measured production cross-sections of Sm and Pt fragments [2] created in the reaction of $1000 \mathrm{~A} \cdot \mathrm{MeV}$ ${ }^{238} \mathrm{U}$ projectiles with a beryllium target in this experiment (black filled circles), shown together with the previous results where a hydrogen target was used (open circles) [15]. The black dashed line represents the predictions of the ABRABLA model [14] and the continuous red line shows the results of COFRA [16] $(Z=73-78)$. The green dotted line shows the prediction of EPAX-3 model [17]. The predictions of EPAX-3 for Sm isotopes is multiplied by a factor of 100 to be visible in this scale.

of neutron-rich nuclei where fragmentation dominates. For the neutron-rich low-Z nuclides EPAX-3 cannot predict the cross sections because fission is not included in the parametrization.

\section{First Measurements with the FRS Ion Catcher}

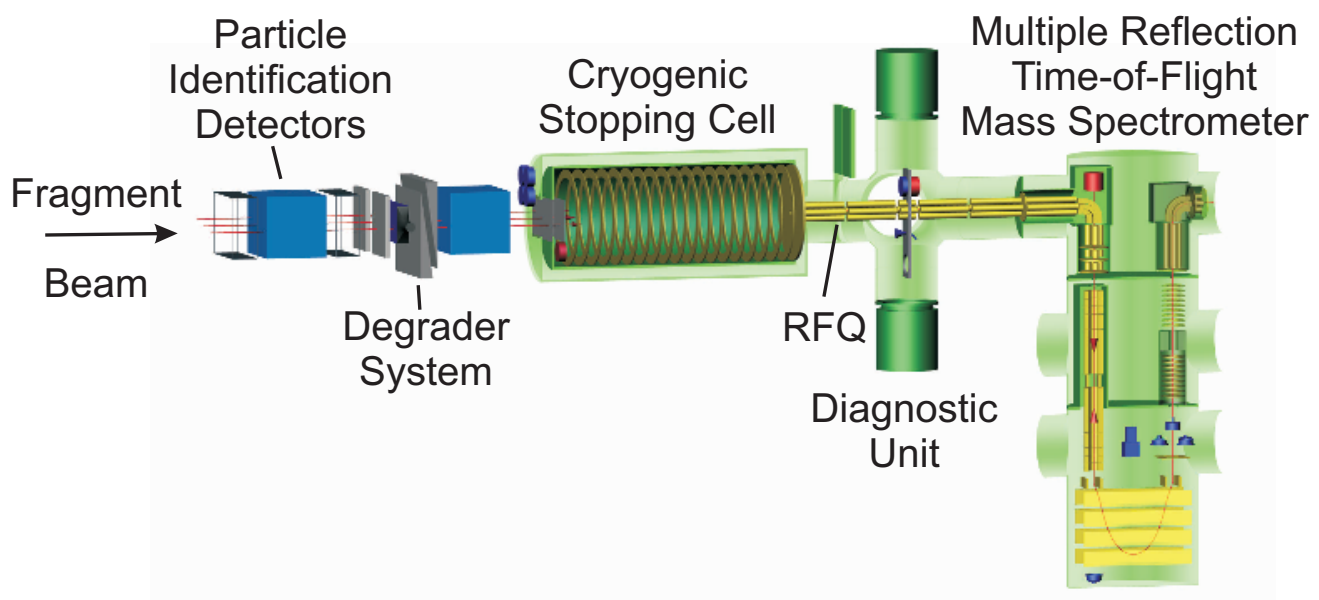

Figure 3. Schematic view of the Cryogenic Stopping Cell (CSC) [8] and the Multiple-Reflection Time-of-Flight Mass Spectrometer (MR-TOF-MS) [21] both were recently commissioned with uranium projectile fragments produced at $1000 \mathrm{~A} \cdot \mathrm{MeV}$ with the FRS. 
The high resolution of the FRS is also required to bunch the momentum distribution such that the separated exotic nuclides can be implanted in thin layers of matter. A special implantation device is a gas-filled Cryogenic Stopping Cell (CSC) [18] characterized by an areal density of a few $\mathrm{mg} / \mathrm{cm}^{2}$ pure He gas. This extremely-small thickness represents a challenge for the energy compression of relativistic fragments with a monoenergetic degrader [19]. The goal is to stop effectively the isotopically separated exotic nuclei in the He gas volume. In the first experiments with the CSC [20] with uranium projectile fragments produced at $1000 \mathrm{~A} \cdot \mathrm{MeV}$, the stopping efficiency after range-bunching with a monoenergetic degrader system placed at the central focal plane of the FRS was about $25 \%$. This experimental result is deduced from range measurements of ${ }^{223} \mathrm{Th}$ fragments. Including the survival and extraction efficiency, the total efficiency of the CSC in the described pioneering experiments was about $10 \%$. Besides the stopping and extraction efficiencies, the extraction time from the CSC is another key parameter. The main goal is to access with the system rare short-lived isotopes. The measured extraction time with ${ }^{221} \mathrm{Ac}$ ions was a few tens of ms. These experimental results are very promising and clearly show the potential of the CSC for the investigation of short-lived exotic nuclides which cannot be accessed by other experimental techniques. The third main component of the experiment, the multiple-reflection time-of-flight mass spectrometer (MR-TOF-MS) [21] has been also applied for the first time to projectile fragments. The extracted ions from the CSC have been mass analyzed with the MR-TOF-MS with the first goal to characterize the performance of the CSC by recording a broad-band mass spectrum. In the second step high-accuracy mass measurements $\left(\Delta \mathrm{m} / \mathrm{m} \approx 10^{-7}\right)$ of short-lived isobars with mass number of $\mathrm{A}=211$ and $\mathrm{A}=213$ have been achieved.

\section{New Mass and Lifetime Measurements of stored Exotic Nuclei}

Uranium fragments were separated in flight with the FRS and injected into the storage-cooler ring ESR [22]. Electron cooling was applied to the stored ions. This forces the circulating ions to the same mean velocity, which is determined by the chosen terminal voltage of the electron cooler. In the present experiment, the velocity of the ions was about $70 \%$ of the velocity of light, corresponding to kinetic energies in the range of (360 to 400) A.MeV. After electron cooling, the velocity spread of low intensity stored fragments amounted to approximately $5 \times 10^{-7}$ [23]. The mass and lifetime values are deduced from the revolution frequencies of the stored and cooled ions with Schottky noise analysis [24]. Schottky Mass Spectrometry (SMS) is able to record many different ion species simultaneously, including nuclides with known masses and nuclides with hitherto unknown masses. SMS is sensitive down to single ions [24] which gives the advantage to resolve in an elegant way ground and low-lying isomeric states. In this experiment [23] we have obtained accurate new mass values of 33 neutron-rich nuclei in the element range from platinum to uranium. A novel data analysis has been applied which reduces the systematic errors to about $10 \mathrm{keV}$ by taking into account the velocity profile of the cooler electrons and the residual ion-optical dispersion in this part of the storage ring.

For very short-lived nuclei cooling would take too long, therefore, isochronous mass spectrometry (IMS) [25] has been performed. IMS measurements are difficult because of the restricted isochronicity range with respect to the different magnetic rigidities of the circulating ions [26, 27]. In addition, the stored ions have different mean velocities and the assignment of reference masses have to take this into account. In a first step to cope with this challenge, we have used the high ion-optical resolution of the FRS to restrict the $\mathrm{B} \rho$ values to $2 \times 10^{-4}$ of the injected ions with special mechanical slits at F2. At FAIR the independent $\mathrm{B} \rho$ determination is planned without restriction of the incident phase-space by the use of additional ToF detectors in the storage ring [28].

A representative comparison of our new experimental SMS and IMS mass values to the widelyused and most accurate macroscopic-microscopic model FRDM [31], the semi-empirical complex 
mass formula of Duflo and Zuker [32], and two microscopic Hartree-Fock-Bogoliubov theories, namely HFB-14 and HFB-17 [33] is shown in Figure 4.
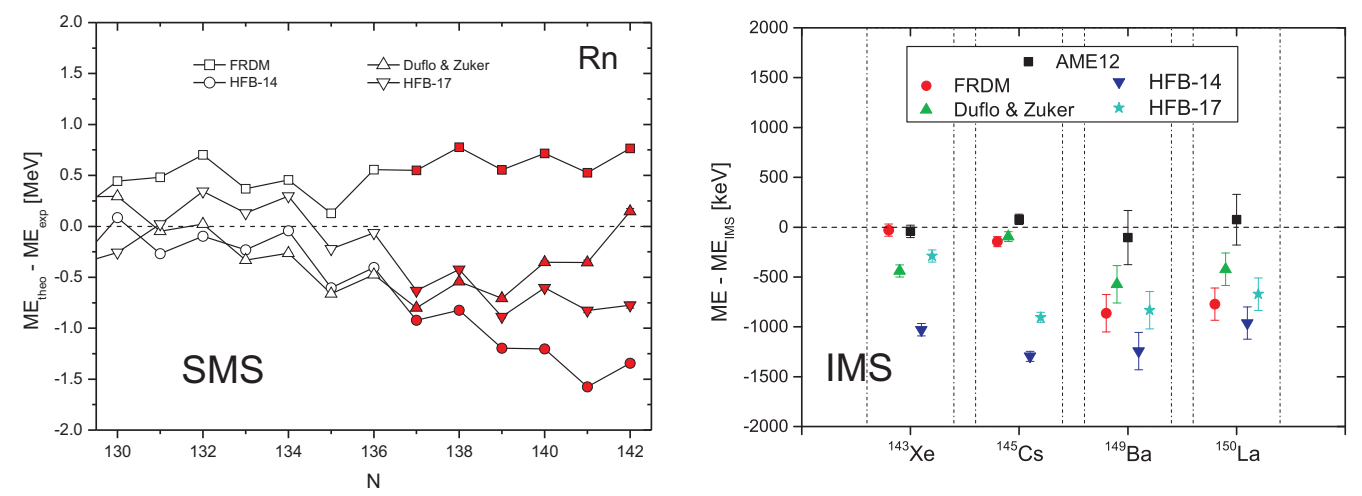

Figure 4. Comparison of experimental Mass Excess (ME) data with different theoretical models [31], [32], [33]. Left panel: SMS results for Rn isotopes [23]. The experimental data are taken from ref. [29] and the present experiments. Right panel: Preliminary representative results for different isotopes from IMS. The masses of ${ }^{149} \mathrm{Ba}$ and ${ }^{150} \mathrm{La}$ have been measured for the first time. For the IMS data the new Atomic Mass Evaluation 2012 is included [30].

The comparison clearly shows deficiencies of the models. The deviations from the experimental data systematically increase with increasing neutron number, i.e., with the distance from the previous experimentally known mass values. Presently, our mass measurements have an order of magnitude smaller uncertainties than theoretical models.

From the pioneering experiments onwards, we performed also lifetime measurements of the stored exotic nuclei. Depending on the magnetic rigidity difference we can record the mother and daughter ions simultaneously in the frequency spectra. The area of the corresponding frequency peaks can yield a first half-life determination, or, owing to the high sensitivity of the Schottky probes, especially the new resonant probe [34], enable decay measurements with a few mother ions and the direct counting of the appearance of the daughter ions separated in the frequency spectrum by the Q-value of the reaction. In this way we discovered new features in the orbital electron capture decay of $\mathrm{H}$ - and He-like ${ }^{140} \mathrm{Pr},{ }^{140} \mathrm{Pm}$ and ${ }^{122} \mathrm{I}[35,36]$.

The experimental results have been explained by Patyk et al. with the extension of the previously existing theory [38]. Our recent lifetime measurements [37] had the goal to investigate a possible difference in the $\alpha$-decay half-life of neutral and $\mathrm{H}$-like ${ }^{213} \mathrm{Fr}$ fragments. The measurement with neutral ions was performed at the F4 focal plane of the FRS via implantation of separated fragments in a position-sensitive silicon detector. The H-like ions were separated and injected into the ESR. The preliminary results from both experiments, $34.03 \pm 0.27$ and $34.4 \pm 1.6 \mathrm{~s}$ for neutral and H-like ions, respectively, have demonstrated that the half-lives agree within the experimental errors. The storage ring experiment will be improved in forthcoming experiments, but already this pilot experiment demonstrates that previous theoretical work which predict a difference of about $40 \%$ cannot be confirmed.

All experiments also have a goal to prepare the experimental setups for the future NUSTAR facility at FAIR. In this spirit, as mentioned above, we have tested the combination of the cryogenic gasstopping cell and multiple-reflection time-of-flight mass spectrometer [20]. 


\section{Super-FRS the Next-Generation In-Flight Facility at FAIR}

Presently, the heavy-ion synchrotron SIS-18 is rather limited in intensity, as regards relativistic heavyion beams, due to space-charge effects. A direct solution is to arrange to accelerate the heaviest projectiles in lower charge states and increase simultaneously the maximum magnetic rigidity of the synchrotron. This idea is elegantly applied by the future project FAIR by upgrading the UNILAC and adding a 100 Tm synchrotron, SIS-100.

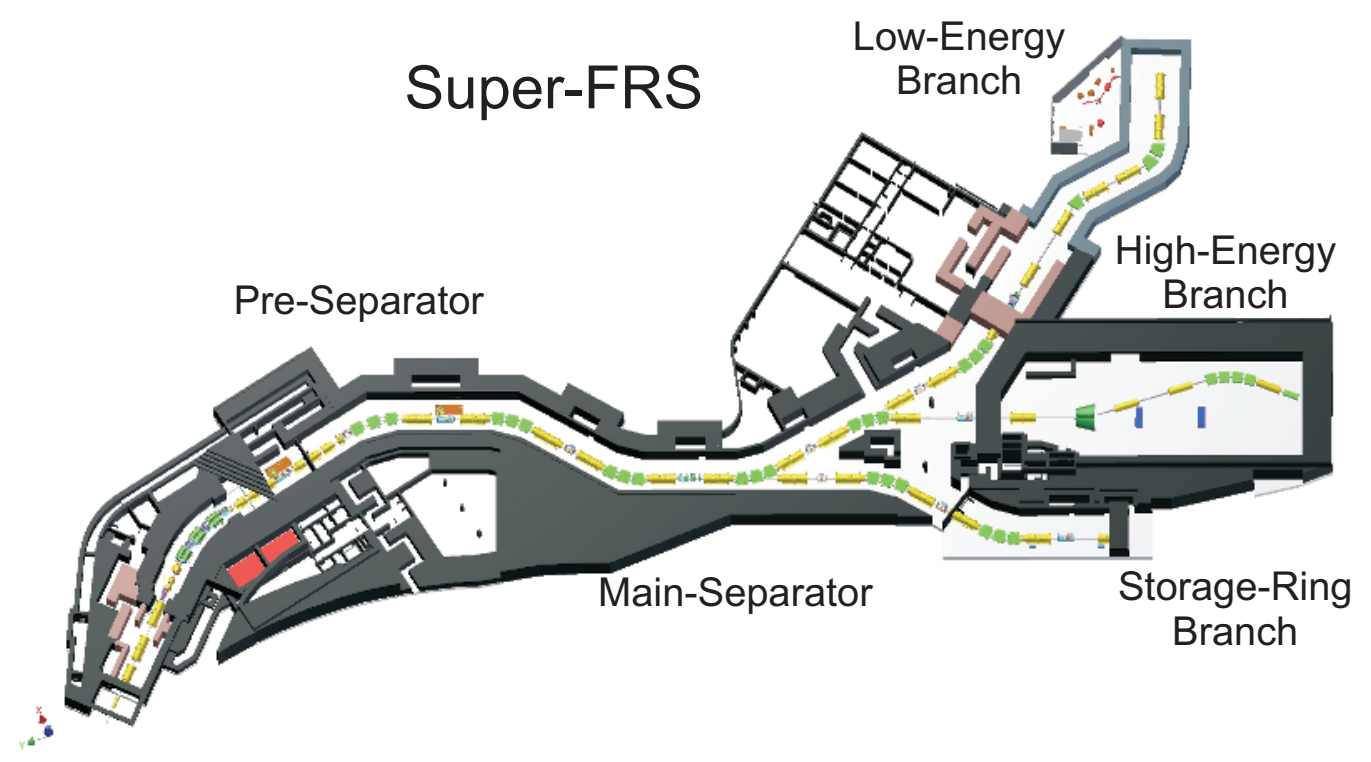

Figure 5. The in-flight separator Super-FRS is the central facility for all NUSTAR experiments which will be performed with the magnet systems directly as Spectrometer Experiments or at the focal planes in the different branches.

The Super-FRS [39] will be the most powerful in-flight separator for exotic nuclei up to relativistic energies corresponding to $20 \mathrm{Tm}$. It is a large-acceptance superconducting fragment separator with three branches serving different experimental areas including a new storage ring complex. The layout of the Super-FRS is shown in Figure 5.

The intensity gain for exotic nuclei compared to the present FRS is mainly for fragment beams with a large phase-space population, such as fission fragments or projectile fragments far off the mass from the primary beam. A simulation with MOCADI using the ion-optical performance of the SuperFRS shows this gain factor for interesting exotic nuclides, see Fig. 6.

In the Low-Energy Branch an Energy Buncher (EB) system [40], consisting of dipole and multipole magnets, will be installed to reduce the energy spread of the fragment beam to enable an efficient stopping in a gas-filled CSC. For this task the ion-optical system of the EB must be per se a highresolution dispersive device. Therefore, the Super-FRS can also be used as a high-resolution spectrometer, in particular when the Main-Separator is dispersion matched with the energy buncher in the Low-Energy Branch. Precise momentum measurements can be performed after secondary reactions, such as nucleon-removal collisions, independent of the large initial momentum spread caused in the production target. 
Fission

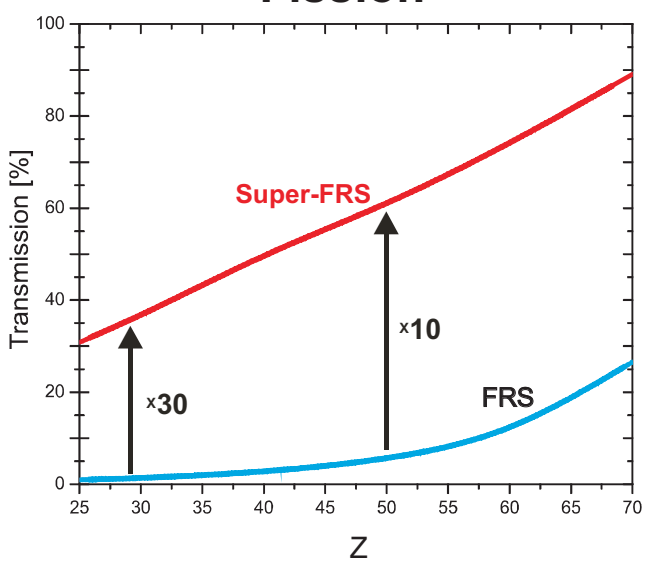

Fragmentation

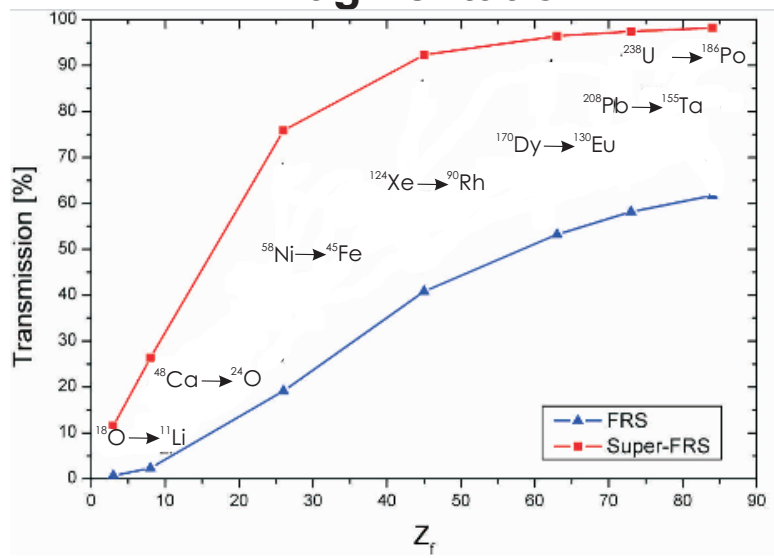

Figure 6. Intensity gain with the large acceptance of the Super-FRS compared to the present FRS. Both for fission fragments and projectile fragments roughly one order of magnitude can be gained for reaction products with large phase space.

\section{Acknowledgement}

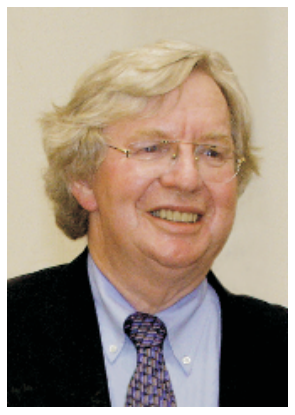

A special wish of the authors is to dedicate this contribution to the late colleague and friend Prof. Dr. Paul Kienle who has strongly supported the construction of the facilities described here and has personally taken part as a driving force in many pioneering experiments at the fragment separator FRS and the combination with the storage-cooler ring ESR. His spirit, ideas and enthusiasm also has been a guide for many young scientists. In this sense he will continue to be present also in future experiments.

\section{References}

[1] K. Langanke, G. Martinez-Pinedo, Rev. Mod. Phys. 758192003.

[2] J. Kurcewicz et al., Phys. Lett. B 717 (2012) 371.

[3] Paul Kienle, Naturwissenschaften 88 (2001) 313, DOI 10.1007/s001140100231

[4] B. Franzke, H. Geissel, G. Münzenberg, Mass Spectro. Rev. 27 (2008) 428.

[5] Y. A. Litvinov and F. Bosch, Rep. Prog. Phys. 74 (2011) 016301.

[6] M. Wada et al. Nucl. Instr. Meth. B 204 (2003) 570.

[7] G. Savard et al., Nucl. Inst. Meth. B 204 (2003) 582.

[8] S. Purushothaman et al., Nucl. Instr. Meth. B 266 (2008) 4488.

[9] J.S. Winfield et al., Nucl. Instrum. Meth. A 704 (2013) 76. 
[10] H. Geissel et al., Nucl. Inst. Meth. B in print (2013), http://dx.doi.org/10.1016/j.nimb.2013.07.064

[11] H. Geissel, et al., Nucl. Instr. Meth. B 70 (1992) 286.

[12] C. Scheidenberger, et al., Nucl. Instr. Meth. B 142 (1998) 441.

[13] N. Iwasa et al., Nucl. Instr. Meth. B 126 (1997) 284.

[14] A. Kelić, M.V. Ricciardi, K.-H. Schmidt IAEA INDC(NDS)-530 (2008) 181-221.

[15] M. Bernas, et al., Nucl. Phys. A 765 (2006) 197.

[16] J. Benlliure et al., Nucl. Phys. A 660 (1999) 87.

[17] K. Sümmerer Phys. Rev. C 86 (2012) 014601.

[18] M. Ranjan et al., Europhys. Lett. 96 (2011) 52001.

[19] H. Geissel, et al., Nucl. Instr. Meth. A 282 (1989) 247.

[20] W. R. Plaß et al., Nucl. Inst. Meth. B in print (2013), http://dx.doi.org/10.1016/j.nimb.2013.07.063.

[21] W. R. Plaß et al., Nucl. Instr. Meth. B 266 (2008) 4560.

[22] B. Franzke, Nucl. Instr. Meth. B 24-25 (1987) 18.

[23] L. Chen et al., Nucl. Phys. A 882 (2012) 71.

[24] Yu.A.Litvinov, et al., Nucl.Phys.A 756 (2005) 3.

[25] M. Hausmann et al., Nucl. Instr. Meth. A 446 (2000) 569.

[26] H. Geissel, et al., Hyp. Int. 173 (2006) 173.

[27] H. Geissel, et al., Eur. Phys. J. Special Topics 150 (2007) 109.

[28] http://www.fair-center.eu/for-users/experiments/nustar/experiments/ilima.html

[29] A. H. Wapstra, G. Audi, C. Thibault, Nucl. Phy. A 729 (2003) 129.

[30] G. Audi, et al. Chin. Phys. C 36 (2012) 1287.

[31] P. Möller et al., At. Data Nucl. Data Tables 59 (1995) 185.

[32] J. Duflo, A.P. Zuker, Phys. Rev. C 52 (1995) R23.

[33] S. Goriely et al., Eur. Phys. J. A 42 (2009) 547;

[34] F. Nolden, et al., Nucl. Instr. Meth. A 659(2011)69.

[35] Yu. A. Litvinov, et al., Phys. Rev. Lett. 99 (2007) 262501.

[36] N. Winckler et al., Phys. Lett. B 679 (2009) 36.

[37] F. Farinon, Ph.D thesis, II. Phys. Inst., Justus Liebig University Gießen 2011.

[38] Z. Patyk, et al., Phys. Rev. C 77 (2008) 014306.

[39] H. Geissel et al., Nucl. Instr. B 204 (2003) 71.

[40] J.S. Winfield et al., Nucl. Instrum. Methods A 704 (2013) 76. 
